\title{
Analysis of fractional COVID-19 epidemic model under Caputo operator
}

\author{
Rahat Zarin ${ }^{1}$, Amir Khan², Abdullahi Yusuf ${ }^{3}$, Mustafa Inc $^{4}$, and Siraj ul-Islam ${ }^{5}$ \\ ${ }^{1}$ University of Engineering \& Technology Department of Chemical Engineering Peshawar \\ Campus \\ ${ }^{2}$ Khyber Pakhtunkhwa Judicial Academy \\ ${ }^{3}$ Biruni University \\ ${ }^{4}$ Firat University \\ ${ }^{5}$ University of Engineering and Technology
}

September 9, 2020

\begin{abstract}
The dynamic of fractional covid-19 epidemic model with a convex incidence rate is studied in this article. Under Caputo operator, existence and uniqueness for the solutions of the fractional covid-19 epidemic model have been ana- lyzed using xed point theorems. We study all the basic properties and results including local and global stability. We show the global stability of disease free equilibrium using the method of Lyapunov function theory while for disease endemic, we use the method of geometrical approach. Moreover, sensitivity analysis complemented by simulations are performed to determine how changes in parameters affect the dynamical behavior of the system.
\end{abstract}

\section{Hosted file}

main.pdf available at https://authorea.com/users/357506/articles/480024-analysis-offractional-covid-19-epidemic-model-under-caputo-operator 
figures/1/1-eps-converted-to.pdf 
figures/2/2-eps-converted-to.pdf 
figures/3/3-eps-converted-to.pdf 
figures/4/4-eps-converted-to.pdf 
figures/5/5-eps-converted-to.pdf 
figures/figure1/figure1-eps-converted-to.pdf 
figures/figure2/figure2-eps-converted-to.pdf 
figures/figure3/figure3-eps-converted-to.pdf 
figures/figure4/figure4-eps-converted-to.pdf 
figures/figure5/figure5-eps-converted-to.pdf 
figures/figure6/figure6-eps-converted-to.pdf 
figures/figure7/figure7-eps-converted-to.pdf 
figures/figure8/figure8-eps-converted-to.pdf 
figures/figure9/figure9-eps-converted-to.pdf 
figures/figure10/figure10-eps-converted-to.pdf 
figures/figure11/figure11-eps-converted-to.pdf 
figures/figure12/figure12-eps-converted-to.pdf 
figures/figure13/figure13-eps-converted-to.pdf 
figures/figure14/figure14-eps-converted-to.pdf 